\title{
Delivering an Interprofessional and Intercollegiate Program be- tween Nursing and Pharmacy Students
}

\author{
Michele S Bednarzyk ${ }^{1}$, Carol Motycka ${ }^{2 *}$ and Kathaleen C Bloom ${ }^{1}$ \\ ${ }^{1}$ School of Nursing, University of North Florida, USA \\ ${ }^{2}$ College of Pharmacy, University of Florida, USA
}

*Corresponding author: Carol Motycka, Pharm.D., BCACP, Clinical Associate Professor, Assistant Dean and Campus Director, College of Pharmacy, University of Florida, Jacksonville Campus, 580 West 8th Street Jacksonville, FL 32209, USA, Tel: 904-244-9590, E-mail: Motycka@cop.ufl.edu

\begin{abstract}
It is clear to many leading national organizations, such as the Institute of Medicine and other professional groups and accrediting bodies that learning in teams with other health professionals is an important way to improve the health and safety of the population. In response to these mandates, many interprofessional health education programs have been developed within universities with an academic medical center. Creating interprofessional educational programs is a challenge for nursing programs in universities that do not have a medical center. The program described here is an innovative teaching-learning approach which is both interprofessional and intercollegiate in nature. Participants include graduate students in nursing at a southeastern university without an academic medical center as well as second year pharmacy students from another southeastern university with academic medical center. The purpose of the program is to foster strong relationships and partnerships among health care providers to maximize the quality of patient care.
\end{abstract}

The concepts underlying interprofessional education and practice are hardly new. A report of a conference on interrelationships of educational programs for health professions [1] called for both interdisciplinary education and interdisciplinary patient care. The recommendations included development of methods to link education with practice realities, and development and use of educational models of interprofessional collaboration in the classroom and in the clinical area. Two IOM reports on quality and safety in the early part of this century: "To Err is Human" [2], and "Crossing the
Quality Chasm" [3], gave rise to IOM's second summit on the education of health professionals [4]. At this summit, five competencies were identified as essential in the education of all health care professionals:

- Patient-centered care;

- Quality improvement;

- Evidence-based practice;

- Informatics; and

- Interdisciplinary teams.

In 2009, the Interprofessional Education Collaborative (IPEC) was formed by members of the America Association of Colleges of Nursing, the American Association of College of Osteopathic Medicine, the American Association of Colleges of Pharmacy, the American Dental Education Association, the Association of Medical Colleges, and the Association of Schools of Public Health [5]. The purpose of the collaborative is to promote interprofessional learning experiences for health professional students. IPEC appointed an expert panel with two members from each organization who were charged with development of recommendations for (a) A core set of competencies for interprofessional collaborative practice, and (b) Educational practices to facilitate for achievement of the competencies. The expert panel identified four competency domains:

- Values/ethics, roles/responsibilities for interprofessional practice;

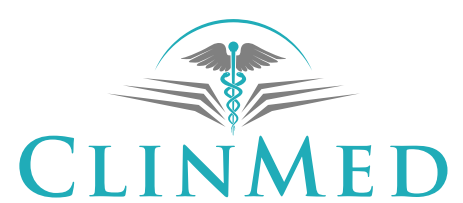

INTERNATIONAL LIBRARY

Citation: Bednarzyk MS, Motycka C, Bloom KC (2017) Delivering an Interprofessional and Intercollegiate Program between Nursing and Pharmacy Students. Int Arch Nurs Health Care 3:075. doi. org/10.23937/2469-5823/1510075

Received: October 26, 2016: Accepted: July 17, 2017: Published: July 20, 2017

Copyright: (c) 2017 Bednarzyk MS, et al. This is an open-access article distributed under the terms of the Creative Commons Attribution License, which permits unrestricted use, distribution, and reproduction in any medium, provided the original author and source are credited. 
- Roles/responsibilities;

- Interprofessional communication; and

- Teams and teamwork.

Interprofessional Education (IPE) has been well-documented in the literature and there is clear evidence that that effective IPE leads to better collaboration in clinical practice $[6,7]$. Potential barriers to IPE include (a) Professional culture and stereotypes, (b) Inconsistent use and different understandings of language, (c) Accreditation and curricula, and (d) Knowledge of roles and scope of other health professions, with professional culture and stereotypes being the most frequently cited barrier. Facilitators of IPE include (a) The presence of leaders and champions, (b) Administrative and institutional support, (c) Mentorship and learning, and (d) Shared vision or mission [7].

In their analysis of IPE across the globe, WHO [6] identified several mechanisms that shape effective IPE, including a resolve to change culture and attitudes of students and providers, willingness to revise existing curricula, and appropriate legislation that eliminates barriers to collaborative practice. Many interprofessional health education programs have been developed within universities with an academic medical center. Creating interprofessional educational programs is a challenge for nursing programs in universities that do not have a medical center.

\section{The Interprofessional, Intercollegiate Education Program}

The program described here is an innovative teaching-learning approach which is both interprofessional and intercollegiate in nature. The purpose of the program is to foster strong relationships and partnerships among three groups of health care providers to maximize the quality of patient care. The program is also designed to provide intercollegiate opportunities for students to engage in interprofessional collaboration. The program has been presented annually for four years.

Participants include first year nurse practitioner and nurse anesthesia students at a southeastern university without an academic medical center as well as second year pharmacy students from another southeastern university with an academic medical center. Faculty include representatives from all programs and universities in which the participants are enrolled.

Three modules are implemented across two semesters. These modules are adaptations of modules developed by faculty in the University of Florida's Office of Interprofessional Education for their InterProfessional Learning in Healthcare course and are co-facilitated by faculty from that office. The modules include all elements of the four interprofessional education competency domains [5] centered on safety, ethics, and determinants of health.
In each module, students first participate in self-study using directed readings and online learning activities. Students then participate in face-to-face activities, including individual activities and interprofessional group solutions for problems related to the content areas using a case study approach. In the interactive sessions, students are placed in teams of 6-8 people, representing all of the disciplines. Groups are rearranged for each of the sessions to allow for differences in experiences. Students begin the interactive sessions by independently completing an Individual Readiness Assessment Test (IRAT) that measures how well they prepared for the session individually. The team then completes a Team Readiness Assessment Test (TRAT) that helps the student team learn how collaboration can increase the collective knowledge among team members. Faculty in the various disciplines facilitate interpersonal and communication skills between the students as they complete their group-work representing different health-care providers.

\section{Module one: Barriers to quality}

Module one focuses on creating a culture of safety. Individual learning activities include completion of three lessons from the Institute for Healthcare Improvement Open School's Quality Improvement (QI) 101: Fundamentals of Improvement course (http://www.ihi.org/ education/ihiopenschool/Courses/Pages/default.aspx). The required lessons are: Errors Can Happen anywhere and to anyone; Health Care Today; and the Institute of Medicine's Aims for Improvement.

During the group session, teams apply the content learned from these individual activities to a given problem scenario. After completion of the IRAT and TRAT, two quality experts guide the teams in evaluating a problem scenario. Each team has a different scenario. Teams are asked to:

- Define the problem depicted in the scenario;

- Identify barriers to quality within the scenario;

- Identify broad QI strategies;

- Develop an action plan consisting of prioritizing the strategies and identifying the most important first step to be taken in solving the problem;

- Present their action plan to the whole group; and

- The day is completed with a debriefing by one of the quality experts who discusses the real world approach to the problem including cost issues and other barriers to the suggestions proposed by students.

\section{Module two: Ethics}

Module two focuses on creating a shared understanding of ethical codes and ethical dilemmas in clinical practice. Individual learning activities include completion of required readings on the ethical code of their 
discipline and on the bioethical principles of respect for autonomy, nonmaleficence, beneficence, and justice. Students are also asked to view a 21-minute film on the Tuskegee experiment. Students then complete an IRAT prior to attending the interactive session.

During the group session, teams discuss their answers on the IRAT and complete the TRAT together, then share their answers with the entire group, engaging in discussion with other groups about their rationale. A faculty expert in ethics provides a brief overview of the bioethical principles and professional codes. Teams are asked to apply the content learned to a given problem scenario. Each team receives a different scenario. Teams are asked to:

- Identify the primary ethical problem depicted in the scenario;

- Specify the professional codes and bioethical principles that are violate;

- Determine the next action steps; and

- Present their decisions and rationale to the whole group.

\section{Module three: Health systems and inequalities}

Module three focuses on creating a shared understanding of social and health-level system factors affecting determinants of health. Individual learning activities include watching "Unnatural Causes... Is Inequality Making Us Sick," a four-hour documentary series exploring racial and socioeconomic inequalities in health (http://www.pbs.org/unnaturalcauses/). Students are also asked to read the associated discussion guide.

During the group session, teams apply the content learned from these individual activities to a given problem scenario. After completion of an IRAT and TRAT, two quality experts guide the teams in evaluating the scenario. Each team has a different scenario. Teams are asked to:

- Identify the problem depicted in the scenario;

- Discuss the disease;

- Discuss factors that increase health disparities generally and impact care;

- Discuss other individual and community-level factors impacting the problem;

- Identify broad strategies to increase care and decrease disparities;

- Develop an action plan consisting of prioritizing the strategies and identifying the most important first step to be taken in solving the problem and the strategy with the most potential impact;

- Present their action plan to the whole group; and

- The day is again completed with a debriefing by one of the quality experts who discusses the real world approach to the problem including cost issues and other barriers to the suggestions proposed by students.

\section{Evaluation of the Program}

\section{Student participation}

Two sessions are completed in the one semester of study and the last one is in the following semester. Both family nurse practitioner students and second year pharmacy students are required to participate in all three modules as part of their coursework. Within the courses, students are only graded on participation with a pass/fail grade given for attending and participating and not the material presented. Due to scheduling conflicts, participation by nurse anesthesia students has been limited to the first two sessions.

The nursing and pharmacy students evaluate the sessions at the end of the semester as a part of the course evaluation. Specific comments about the sessions are generally positive.

\section{Facilitators}

Previously identified facilitators of interprofessional education include shared vision, leaders and champions, mentorship and learning, and administrative and institutional support $[7,8]$. The program described here enjoyed elements of all of these facilitators. Certainly there has been a shared vision between faculty champions of all four programs and at both universities. Collaborative planning begins early and all faculty and group facilitators remain flexible and attempt meet the changing needs of students and programs. The education and mentorship provided by personnel from the Office of Interprofessional Education at the University of Florida have been invaluable to the success of the program, as has the administrative support from the University of North Florida School of Nursing and the University of Florida College of Pharmacy.

\section{Barriers}

Similar to what has been found in most previous studies, the largest barriers encountered were institutional in nature [8]. Scheduling was a particular challenge. With several programs and two universities included, finding a date and time that worked for everyone, including travel time, was difficult. The sessions took 3-4 hours to complete, plus the time taken to commute and park, which added stress to already full schedules. From the pharmacy students' perspective, driving to another university and then finding parking at the other institution was often a challenge. The fact that some of the facilitators also travel from another city added to the scheduling difficulties.

A second institutional barrier had to do with overall commitment to the program on the part of the students 
and the academic programs in which the students were studying. Understanding and valuing interprofessional education and commitment to its implementation varied over the four years of implementation of the program. In the first year, two other graduate level health majors were involved in the planning: physical therapy and nutrition and dietetics. Because of changes in faculty within the programs and changes in the programs themselves, these students participated only marginally in the first year and not at all thereafter.

Participation was required for all pharmacy students as part of their coursework and this requirement was included in the course syllabus. Participation in the first two sessions was required for all nursing students (nurse practitioner and nurse anesthesia) enrolled in an advanced pharmacology course. However, the third session, which occurred the following semester, was only required of the nurse practitioner students. The fact that participation was mandatory, but not graded, may have had an impact on the value the students placed on the process.

\section{Discussion}

The facilitators and barriers found with this program-scheduling and logistics, valuing and commitment on the part of programs, faculty and students-are not unlike those previously described in the literature and synthesized in the review by Lawlis, et al. [8]. The faculty and facilitators continue to work to overcome or at least minimize the identified barriers. Faculty are also exploring the feasibility and logistics of expanding the program to include additional professional students from these two institutions and potentially others. Barriers identified in the review [8] that were not evident in the program described here include biases, lack of respect, and turf battles. These potential impediments to interprofessional collaboration will be critically appraised as the program expands to include additional professionals.

"Many health science professionals have been exposed to a monochromatic view of education that is lecture-based and behavioristically driven," said Erik Black, Ph.D., an assistant professor in the College of Medicine department of pediatrics and the College of Education School of Teaching and Learning. "That is not necessar- ily where medical education is going. Today, there is a growing emphasis on small group learning, team-based learning and constructivist principles of instruction and learning" [9].

The bottom line is, interprofessional education provides students with the ability to practice shared decision-making, manage conflicts and show flexibility when working with other professionals. Ultimately, joint learning opportunities for students from different disciplines and universities will result in more collaborative practice skills when these students become health professionals, and this, in turn, should help lead to higher quality, safer clinical care.

\section{Acknowledgement}

Erik W. Black, PhD, Assistant Professor in Pediatrics and Educational Technology in the University of Florida College of Medicine.

\section{References}

1. Institute of Medicine (1972) Educating for the health team. National Academy Press, Washington, DC.

2. Institute of Medicine (2000) To err is human: Building a safer health system. National Academy Press, Washington DC.

3. Institute of Medicine (2001) Crossing the quality chasm: A New Health System for the 21st Century. National Academy Press, Washington DC.

4. Institute of Medicine (2003) Health professions education: A bridge to quality. The National Academies Press, Washington, DC.

5. Interprofessional Education Collaborative Expert Panel (2011) Core competencies for interprofessional collaborative practice: Report of an expert panel. Interprofessional Education Collaborative, Washington, D.C.

6. World Health Organization (2010) Framework for action on interprofessional education and collaborative practice. Geneva, Switzerland.

7. World Health Organization (2013) Interprofessional collaborative practice in primary health care: Nursing and midwifery perspectives: six case studies. Human Resources for Health Observer.

8. Lawlis TR, Anson J, Greenfield D (2014) Barriers and enablers that influence sustainable interprofessional education: a literature review. J Interprof Care 28: 305-310.

9. (2011) UF education, medical colleges team up on new master's degree to help doctors become better teachers. EduGator News. 